\title{
The spaces of formal power series of class $M$ of Roumieu type and of Beurling type
}

\author{
Dedicate to Professor R. Ishimura \\ Xiaoran JIN \\ (Received March 7, 2019) \\ (Revised August 8, 2019)
}

\begin{abstract}
In this article, we introduce the space of formal power series of class $\mathbb{M}$ of Roumieu (resp., Beurling) type, which is the generalization of the space of entire functions of normal (resp., minimal) type with respect to a proximate order. And we characterize continuous endomorphisms of these spaces.
\end{abstract}

\section{Introduction}

In [3] and [4], R. Ishimura proved that any continuous endomorphism of the sheaf or stalks of holomorphic functions has been characterized as a partial differential operator with holomorphic coefficients of infinite order. In [5], $\mathrm{R}$. Ishimura tried to solve the characterization problem of continuous endomorphism for the case of space of entire functions of normal type with respect to a proximate order.

Recently, in [1], Aoki, Ishimura, Okada, Struppa, and Uchida characterized continuous endomorphism of the spaces of entire functions of normal type and minimal type with respect to a given order. Furthermore, in [2], they generalized these conclusions to the case of proximate order.

In the present paper, we introduce the spaces of formal power series of class $\mathbb{M}$ of Roumieu type and of Beurling type. The idea of $\mathbb{M}$ is from [8], so are the name of Roumieu type and of Beurling type. It turns out that these two spaces are more general than all the spaces referred above. We characterize continuous endomorphisms of these spaces. As an application of our main results, we extend the theorems of [2].

\section{Preliminary}

In this article, we employ the same notations as [6]: for a point $z:=$ $\left(z_{1}, \ldots, z_{n}\right) \in \mathbb{C}^{n}$ and for multi-indexes $\alpha=\left(\alpha_{1}, \ldots, \alpha_{n}\right), \beta=\left(\beta_{1}, \ldots, \beta_{n}\right) \in \mathbb{N}^{n}$

2010 Mathematics Subject Classification. 32A05, 32A15, 30D15.

Key words and phrases. formal power seires; Roumieu type; Beurling type. 
with $\mathbb{N}:=\{0,1,2, \ldots\}$, we set

$$
\begin{array}{rlrl}
|z| & :=\sqrt{\left|z_{1}\right|^{2}+\cdots+\left|z_{n}\right|^{2},} & \partial^{\alpha} & :=\partial_{z}^{\alpha}:=\frac{\partial^{|\alpha|}}{\partial z_{1}^{\alpha_{1}} \ldots \partial z_{n}^{\alpha_{n}},} \\
|\vec{z}| & :=\left(\left|z_{1}\right|, \ldots,\left|z_{n}\right|\right), & |\alpha|:=\alpha_{1}+\cdots+\alpha_{n}, \\
\alpha ! & :=\alpha_{1} ! \ldots \alpha_{n} !, & \alpha-\beta & :=\left(\alpha_{1}-\beta_{1}, \ldots, \alpha_{n}-\beta_{n}\right), \\
\left(\begin{array}{c}
\alpha \\
\beta
\end{array}\right) & :=\frac{\alpha !}{(\alpha-\beta) ! \beta !}=\left(\begin{array}{c}
\alpha_{1} \\
\beta_{1}
\end{array}\right) \ldots\left(\begin{array}{c}
\alpha_{n} \\
\beta_{n}
\end{array}\right), & \alpha^{\beta}:=\alpha_{1}^{\beta_{1}} \ldots \alpha_{n}^{\beta_{n}} .
\end{array}
$$

Let $\mathbb{M}:=\left(M_{\alpha}\right)_{\alpha \in \mathbb{N}^{n}}$ be a sequence of positive real numbers with the following property:

(M1) There exist $C, t>1$ such that for all $\alpha, \beta \in \mathbb{N}^{n}$, we have

$$
\max \left\{\frac{M_{\alpha+\beta}}{M_{\alpha} M_{\beta}}, \frac{M_{\alpha} M_{\beta}}{M_{\alpha+\beta}}\right\} \leqslant C t^{|\alpha+\beta|} .
$$

We take a constant $t>1$ that satisfies (M1) and fix it in the sequel.

REMARK 1. Observe that

(i) if $p \in \mathbb{R}$ and $\left(M_{\alpha}\right)_{\alpha \in \mathbb{N}^{n}}$ satisfies (M1), then so does $\left(\left(M_{\alpha}\right)^{p}\right)_{\alpha \in \mathbb{N}^{n}}$;

(ii) if both of $\left(M_{\alpha}\right)_{\alpha \in \mathbb{N}^{n}}$ and $\left(M_{\alpha}^{\prime}\right)_{\alpha \in \mathbb{N}^{n}}$ satisfy (M1), then so does $\left(M_{\alpha} M_{\alpha}^{\prime}\right)_{\alpha \in \mathbb{N}^{n}}$

Definition 1. Suppose that $f(z):=\sum_{\alpha \in \mathbb{N}^{n}} f_{\alpha} z^{\alpha}, r>0$, and that the sequence $\mathbb{I}$ satisfies (M1). We define a subspace of the space of formal power series:

$$
B_{r}^{\mathbb{M}}:=\left\{f(z) \in \mathbb{C}[[z]]\left|\|f(z)\|_{r}:=\sup _{\alpha \in \mathbb{N}^{n}}\right| f_{\alpha} \mid \frac{M_{\alpha}}{r^{|\alpha|}}<\infty\right\},
$$

which is a Banach space with norm $\|\cdot\|_{r}$. Now we consider the space of formal power series of class $\mathbb{M}$ of Roumieu type

$$
\mathscr{F}\{\mathbb{M}\}:=\lim _{r \rightarrow \infty} B_{r}^{\mathbb{M}}
$$

and the space of formal power series of class $\mathbb{M}$ of Beurling type

$$
\mathscr{F}^{(\mathbb{M})}:=\lim _{r \rightarrow 0} B_{r}^{\mathbb{M}} .
$$

We have that $\mathscr{F}^{\{\mathbb{M}\}}$ (resp., $\mathscr{F}^{(\mathbb{M})}$ ) is a (DFS)-space (resp., an (FS)-space), as the following lemma. 
LeMmA 1. If $0<s<r$, then the inclusion mapping $B_{s}^{\mathbb{M}} \hookrightarrow B_{r}^{\mathbb{M}}$ is compact.

Proof. Set $B:=\left\{f \in B_{s}^{\mathbb{M}}:\|f\|_{s} \leqslant 1\right\}$. We need to show that the set $B$ is relatively compact in $B_{r}^{\mathbb{M}}$. Suppose $f^{j}(z) \in B$ for all $j \in \mathbb{N}$, where $f^{j}(z):=$ $\sum_{\alpha \in \mathbb{N}^{n}} f_{\alpha^{j}}^{j} z^{\alpha}$. It suffices to show that there exists an accumulation point $f(z):=\sum_{\alpha \in \mathbb{N}^{n}} f_{\alpha} z^{\alpha}$ of the sequence $\left(f^{j}(z)\right)_{j}$ in $B_{r}^{\mathbb{M}}$.

First, we construct this $f(z)$. For each $\alpha \in \mathbb{N}^{n}$, we have

$$
\left|f_{\alpha}^{j}\right| \leqslant\left\|f^{j}(z)\right\|_{s} \frac{s^{|\alpha|}}{M_{\alpha}} \leqslant \frac{s^{|\alpha|}}{M_{\alpha}}
$$

whenever $j \in \mathbb{N}$. Thus, there exists a subsequence $\left(k_{0}(j)\right)_{j}$ of $\mathbb{N}$ such that $f_{0}^{k_{0}(j)} \rightarrow f_{0}$ as $j \rightarrow \infty$. For the same reason, there exists a subsequence $\left(k_{1}(j)\right)_{j}$ of the sequence $\left(k_{0}(j)\right)_{j}$ such that for each $|\alpha|=1$, we have $f_{\alpha}^{k_{|\alpha|}(j)} \rightarrow f_{\alpha}$ as $j \rightarrow \infty$. Repeating this process, we obtain $f_{\alpha}$ for each $\alpha \in \mathbb{N}$. Set $f(z):=\sum_{\alpha \in \mathbb{N}^{n}} f_{\alpha} z^{\alpha}$.

Now we show that for any $\varepsilon>0$, there exists a subsequence $k(j)$ of $\mathbb{N}$ such that $\left\|f^{k(j)}(z)-f(z)\right\|_{r} \leqslant \varepsilon$ whenever $j \in \mathbb{N}$. Since $r>s$, there exists $N>0$ such that

$$
\left(\frac{s}{r}\right)^{|\alpha|}<\frac{\varepsilon}{4}
$$

whenever $|\alpha|>N$. In view of (2.1), we have $\left|f_{\alpha}\right| \leqslant s^{|\alpha|} / M_{\alpha}$ for all $\alpha \in \mathbb{N}$, which implies that $\|f(z)\|_{s} \leqslant 1$. Thus, for all $j \in \mathbb{N}$, we have that

$$
\begin{aligned}
\left\|\sum_{|\alpha|>N}\left(f_{\alpha}^{j}-f_{\alpha}\right) z^{\alpha}\right\|_{r} & =\sup _{|\alpha|>N}\left|f_{\alpha}^{j}-f_{\alpha}\right| \frac{M_{\alpha}}{r^{|\alpha|}} \\
& \leqslant \sup _{|\alpha|>N}\left|f_{\alpha}^{j}\right| \frac{M_{\alpha}}{S^{|\alpha|}}\left(\frac{s}{r}\right)^{|\alpha|}+\sup _{|\alpha|>N}\left|f_{\alpha}\right| \frac{M_{\alpha}}{S^{|\alpha|}}\left(\frac{s}{r}\right)^{|\alpha|} \\
& \leqslant\left\|f^{j}(z)\right\|_{s} \frac{\varepsilon}{4}+\|f(z)\|_{s} \frac{\varepsilon}{4} \leqslant \frac{\varepsilon}{2} .
\end{aligned}
$$

On the other hand, by the construction of the sequence $\left(k_{N}(j)\right)_{j}$, there exists $J \in \mathbb{N}$ such that

$$
\left|f_{\alpha}^{k_{N}(j)}-f_{\alpha}\right| \leqslant \frac{\varepsilon}{2} \min _{|\beta| \leqslant N} \frac{r^{|\beta|}}{M_{\beta}}
$$

whenever $j>J$ and $|\alpha| \leqslant N$. By (2.2) and (2.3), we have 


$$
\begin{aligned}
\left\|f^{k_{N}(j)}(z)-f(z)\right\|_{r} & =\left\|\sum_{|\alpha| \geqslant 0}\left(f_{\alpha}^{k_{N}(j)}-f_{\alpha}\right) z^{\alpha}\right\|_{r} \\
& \leqslant\left\|\sum_{|\alpha| \leqslant N}\left(f_{\alpha}^{k_{N}(j)}-f_{\alpha}\right) z^{\alpha}\right\|_{r}+\left\|\sum_{|\alpha|>N}\left(f_{\alpha}^{k_{N}(j)}-f_{\alpha}\right) z^{\alpha}\right\|_{r} \\
& \leqslant \max _{|\alpha| \leqslant N}\left(\frac{\varepsilon}{2} \min _{|\beta| \leqslant N} \frac{r^{|\beta|}}{M_{\beta}}\right) \frac{M_{\alpha}}{r^{|\alpha|}}+\frac{\varepsilon}{2}=\varepsilon
\end{aligned}
$$

whenever $j>J$. Therefore, we see that $f(z)$ is an accumulation point of the sequence $\left(f^{j}(z)\right)_{j}$ in $B_{r}^{\mathbb{M}}$, as desired.

By the definitions of $\mathscr{F}^{\{\mathbb{M}\}}$ and $\mathscr{F}(\mathbb{M})$, we have the following propositions immediately.

Proposition 1. Suppose that $f(z)=\sum_{\alpha \in \mathbb{N}^{n}} f_{\alpha} z^{\alpha}$ is a formal power series.

(1) $f(z)$ belongs to $\mathscr{F}\{\mathbb{M}\}$ if and only if we have that

$$
\limsup _{|\alpha| \rightarrow \infty}\left(\left|f_{\alpha}\right| M_{\alpha}\right)^{1 /|\alpha|}<\infty .
$$

(2) $f(z)$ belongs to $\mathscr{F}^{(\mathbb{M})}$ if and only if we have that

$$
\limsup _{|\alpha| \rightarrow \infty}\left(\left|f_{\alpha}\right| M_{\alpha}\right)^{1 /|\alpha|}=0 .
$$

By the binomial theorem, for all $\alpha, \beta \in \mathbb{N}^{n}$, we have

$$
1 \leqslant \frac{(\alpha+\beta) !}{\alpha ! \beta !}=\left(\begin{array}{c}
\alpha+\beta \\
\alpha
\end{array}\right) \leqslant \sum_{\lambda \leqslant \alpha+\beta}\left(\begin{array}{c}
\alpha+\beta \\
\lambda
\end{array}\right)=2^{|\alpha+\beta|},
$$

which means that $(\alpha !)_{\alpha \in \mathbb{N}^{n}}$ satisfies (M1). By Remark 1 (i), in the following cases, the sequence $\left(M_{\alpha}\right)_{\alpha \in \mathbb{N}^{n}}$ satisfies (M1):

(1) $M_{\alpha}:=(\alpha !)^{1 / \rho}$, where $\rho>0$. In this case, the spaces $\mathscr{F}^{\{\mathbb{M}\}}$ and $\mathscr{F}^{(\mathbb{M})}$ coincide with the spaces studied in [1] (in the sense of topological space), respectively.

(2) $M_{\alpha}:=A_{|\alpha|}$, where $A_{|\alpha|}$ is given by (6.1). In this case, which is the generalization of $(1)$, the spaces $\mathscr{F}^{\{\mathbb{M}\}}$ and $\mathscr{F}^{(\mathbb{M})}$ coincide with the spaces studied in [2] (in the sense of topological space), respectively. In the end of this paper, we will take a close look at this case as an application of our main results.

(3) $M_{\alpha}:=(\alpha !)^{p}$, where $p<0$. This case was first considered by $\mathrm{R}$. Ishimura and studied by $\mathrm{S}$. Tatemichi. 


\section{Formal theory}

The set of all formal differential operators of the form

$$
P:=P\left(z, \partial_{z}\right):=\sum_{\alpha \in \mathbb{N}^{n}} a_{\alpha}(z) \partial_{z}^{\alpha}
$$

is denoted by $\mathscr{D}$, where $a_{\alpha}(z) \in \mathbb{C}[[z]]$. For any $f(z) \in \mathbb{C}[[z]]$ and $v \in \mathbb{N}^{n}$, we set $\partial_{z=0}^{v} f(z):=\partial_{z}^{v} f(0)$. The set of all formal differential operators of the form (in the sequel, we assume $Q$ has the following form)

$$
Q:=Q\left(z, \partial_{z=0}\right):=\sum_{v \in \mathbb{N}^{n}} b_{v}(z) \partial_{z=0}^{v}
$$

is denoted by $\mathscr{L}$, where $b_{v}(z) \in \mathbb{C}[[z]]$. We define the mapping $I$ by

$$
\begin{aligned}
I: \mathscr{D} & \rightarrow \mathscr{L}, \\
& P \mapsto Q:=\sum_{v \in \mathbb{N}^{n}} P\left(\frac{z^{v}}{v !}\right) \partial_{z=0}^{v} .
\end{aligned}
$$

In what follows, we always assume $a_{\alpha}(z)$ and $b_{v}(z)$ have the form:

$$
a_{\alpha}(z):=\sum_{\beta \in \mathbb{N}^{n}} a_{\alpha}^{\beta} z^{\beta} \quad \text { and } \quad b_{v}(z):=\sum_{\mu \in \mathbb{N}^{n}} b_{v}^{\mu} z^{\mu} .
$$

Lemma 2. Let $P \in \mathscr{D}$ and $Q \in \mathscr{L}$. Then the following statements are equivalent:

(1) $I(P)=Q$;

(2) For all $v \in \mathbb{N}^{n}$, we have

$$
b_{v}(z)=P\left(\frac{z^{v}}{v !}\right)=\sum_{\alpha \leqslant v} \frac{z^{v-\alpha}}{(v-\alpha) !} a_{\alpha}(z)
$$

(3) For all $\alpha \in \mathbb{N}^{n}$, we have

$$
a_{\alpha}(z)=\sum_{v \leqslant \alpha} \frac{(-z)^{\alpha-v}}{(\alpha-v) !} b_{v}(z) ;
$$

(4) For all $v, \mu \in \mathbb{N}^{n}$, we have

$$
b_{v}^{\mu}=\sum_{\substack{\lambda \leqslant v \\ \lambda \leqslant \mu}} \frac{a_{v-\lambda}^{\mu-\lambda}}{\lambda !}
$$


(5) For all $v, \mu \in \mathbb{N}^{n}$, we have

$$
a_{v}^{\mu}=\sum_{\substack{\lambda \leqslant v \\ \lambda \leqslant \mu}} \frac{(-1)^{\lambda}}{\lambda !} b_{v-\lambda}^{\mu-\lambda}
$$

Consequently, the mapping $I: \mathscr{D} \rightarrow \mathscr{L}$ is bijective.

Proof. By the definition of the mapping $I$, we see (1) is equivalent to (2). Hence, for each $v \in \mathbb{N}^{n}$, we have

$$
\begin{aligned}
\sum_{\mu \in \mathbb{N}^{n}} b_{v}^{\mu} z^{\mu}=b_{v}(z) & =\sum_{\alpha \leqslant v} \frac{z^{v-\alpha}}{(v-\alpha) !} a_{\alpha}(z) \\
& =\sum_{\alpha \leqslant v} \sum_{\beta \in \mathbb{N}^{n}} \frac{z^{v-\alpha}}{(v-\alpha) !} a_{\alpha}^{\beta} z^{\beta} \\
& =\sum_{\lambda \leqslant v} \sum_{\beta \in \mathbb{N}^{n}} \frac{a_{v-\lambda}^{\beta}}{\lambda !} z^{\beta+\lambda}=\sum_{\lambda \leqslant v} \sum_{\mu \in \mathbb{N}^{n}} \sum_{\lambda \leqslant \mu} \frac{a_{v-\lambda}^{\mu-\lambda}}{\lambda !} z^{\mu},
\end{aligned}
$$

where $\lambda:=v-\alpha$ and $\mu:=\beta+\lambda$. Thus, (2) is equivalent to (4). By the same process, we see (3) is equivalent to (5).

To see (2) implies (3), observe that for each $\gamma<\alpha$, we have

$$
\sum_{\gamma \leqslant v \leqslant \alpha} \frac{(-1)^{|\alpha-v|}}{(\alpha-v) !(v-\gamma) !}=\frac{1}{(\alpha-\gamma) !} \sum_{\gamma \leqslant v \leqslant \alpha}\left(\begin{array}{c}
\alpha-\gamma \\
\alpha-v
\end{array}\right)(-1)^{|\alpha-v|}=0 .
$$

Thus, for each $\alpha \in \mathbb{N}^{n}$, we have

$$
\begin{aligned}
\sum_{v \leqslant \alpha} \frac{(-z)^{\alpha-v}}{(\alpha-v) !} b_{v}(z) & =\sum_{v \leqslant \alpha} \frac{(-z)^{\alpha-v}}{(\alpha-v) !} \sum_{\gamma \leqslant v} \frac{z^{v-\gamma}}{(v-\gamma) !} a_{\gamma}(z) \\
& =\sum_{\gamma \leqslant \alpha} z^{\alpha-\gamma} a_{\gamma}(z) \sum_{\gamma \leqslant v \leqslant \alpha} \frac{(-1)^{|\alpha-v|}}{(\alpha-v) !(v-\gamma) !}=a_{\alpha}(z),
\end{aligned}
$$

as required. By the same process, we see (3) implies (2).

In (2), we see that $b_{v}(z) \in \mathbb{C}[[z]]$ whenever $a_{\alpha}(z) \in \mathbb{C}[[z]]$. Thus, the mapping $I: \mathscr{D} \rightarrow \mathscr{L}$ is well-defined. For the similar reason, (3) yields that the mapping $I$ is surjective. Finally, (5) implies that the mapping $I$ is injective.

For any $P \in \mathscr{D}$ and $f(z):=\sum_{v \in \mathbb{N}^{n}} f_{v} z^{v} \in \mathbb{C}[[z]]$, we have formally

$$
\begin{aligned}
P f & =\sum_{\alpha \in \mathbb{N}^{n}} a_{\alpha}(z) \partial_{z}^{\alpha} f(z) \\
& =\sum_{\alpha \in \mathbb{N}^{n}}\left(\sum_{\beta \in \mathbb{N}^{n}} a_{\alpha}^{\beta} z^{\beta}\right)\left(\sum_{v \geqslant \alpha} f_{v} \frac{v !}{(v-\alpha) !} z^{v-\alpha}\right)=\sum_{\alpha \in \mathbb{N}^{n}} c_{\alpha}(z),
\end{aligned}
$$


where

$$
c_{\alpha}(z):=\sum_{\mu \in \mathbb{N}^{n}}\left(\sum_{\substack{\beta+v-\alpha=\mu \\ v \geqslant \alpha}} a_{\alpha}^{\beta} \frac{v !}{(v-\alpha) !} f_{v}\right) z^{\mu} .
$$

For each $\alpha \in \mathbb{N}^{n}, c_{\alpha}(z)$ is a formal power series. However, for each $\mu \in \mathbb{N}^{n}$, the sum of the coefficients of $z^{\mu}$ in the series $\sum_{\alpha \in \mathbb{N}^{n}} c_{\alpha}(z)$ is not necessarily convergent. So we need the following definition to ensure that $P f$ is not ambiguous.

Definition 2. Suppose $P \in \mathscr{D}$ and $f(z):=\sum_{v \in \mathbb{N}^{n}} f_{v} z^{v} \in \mathbb{C}[[z]]$. We say $P$ is formally well-defined at $f$ provided that for all $\mu \in \mathbb{N}^{n}$, we have

$$
\sum_{v \in \mathbb{N}^{n}} \sum_{\substack{\lambda \leqslant v \\ \lambda \leqslant \mu}}\left|a_{v-\lambda}^{\mu-\lambda} \frac{v !}{\lambda !} f_{v}\right|<\infty .
$$

Let $S$ be a subset of $\mathbb{C}[[z]]$. We say $P$ is formally well-defined on $S$ if $P$ is formally well-defined at any $f \in S$.

Proposition 2. If $P$ is formally well-defined at $f$, then $P f=I(P) f \in$ $\mathbb{C}[[z]]$.

Proof. Since $P$ is formally well-defined at $f$, by Lemma 2, there exists $Q \in \mathscr{L}$ such that $Q=I(P)$, and for all $\mu \in \mathbb{N}^{n}$, we have that

$$
\sum_{v \in \mathbb{N}^{n}}\left|b_{v}^{\mu} v ! f_{v}\right| \leqslant \sum_{v \in \mathbb{N}^{n}} \sum_{\substack{\lambda \leqslant v \\ \lambda \leqslant \mu}}\left|a_{\nu-\lambda}^{\mu-\lambda} \frac{v !}{\lambda !} f_{v}\right|<\infty,
$$

which implies that the series $\sum_{v \in \mathbb{N}^{n}} b_{v}^{\mu} v ! f_{v}$ is absolutely convergent. Hence, setting $\lambda:=v-\alpha$ and $\mu:=\beta+\lambda$, we obtain that

$$
\begin{aligned}
P f & =\sum_{\alpha \in \mathbb{N}^{n}}\left(\sum_{\mu \in \mathbb{N}^{n}}\left(\sum_{\beta+v-\alpha=\mu} a_{\alpha}^{\beta} \frac{v !}{(v-\alpha) !} f_{v}\right) z^{\mu}\right) \\
& =\sum_{\mu \in \mathbb{N}^{n}}\left(\sum_{v \in \mathbb{N}^{n}}\left(\sum_{\substack{\lambda \leqslant v \\
\lambda \leqslant \mu}} a_{v-\lambda}^{\mu-\lambda} \frac{v !}{\lambda !}\right) f_{v}\right) z^{\mu} \\
& =\sum_{\mu \in \mathbb{N}^{n}}\left(\sum_{v \in \mathbb{N}^{n}} b_{v}^{\mu} v ! f_{v}\right) z^{\mu} \\
& =\sum_{v \in \mathbb{N}^{n}} b_{v}(z) v ! f_{v}=\sum_{v \in \mathbb{N}^{n}} b_{v}(z) \partial_{z}^{v} f(0)=I(P) f .
\end{aligned}
$$


It follows that $I(P) f \in \mathbb{C}[[z]]$ from the convergence of the series $\sum_{v \in \mathbb{N}^{n}} b_{v}^{\mu} v ! f_{v}$.

\section{Continuous endomorphisms of $\mathscr{F}\{\mathbb{M}\}$}

Definition 3. Let $\mathbb{M}^{*}:=\left(M_{\alpha}^{*}\right)_{\alpha \in \mathbb{N}^{n}}$, where $M_{\alpha}^{*}:=\alpha ! / M_{\alpha}$. A differential operator $P \in \mathscr{D}$ is of class $\left\{\mathbb{M}^{*}\right\}$ (resp., of class $\left(\mathbb{M}^{*}\right)$ ) provided that for any $\varepsilon>0$, there exist $C, r>0$ (resp., for any $r>0$, there exist $C, \varepsilon>0$ ) such that

$$
\left\|a_{\alpha}(z)\right\|_{r} \leqslant C \frac{\varepsilon^{|\alpha|}}{M_{\alpha}^{*}}
$$

for all $\alpha \in \mathbb{N}^{n}$. The set of all differential operators of class $\left\{\mathbb{M}^{*}\right\}$ (resp., of class $\left.\left(\mathbb{M}^{*}\right)\right)$ is denoted by $\mathscr{D}^{\left\{\mathbb{M}^{*}\right\}}$ (resp., $\mathscr{D}^{\left(\mathbb{M}^{*}\right)}$. For convenience, we also set

$$
\begin{aligned}
\mathscr{L}^{\left\{\mathbb{M}^{*}\right\}}:= & \{Q \in \mathscr{L} \mid \text { for any } \varepsilon>0, \text { there exist } C, r>0 \text { such that } \\
& \left.\left\|b_{v}(z)\right\|_{r} \leqslant C \frac{\varepsilon^{|v|}}{M_{v}^{*}} \text { for all } v \in \mathbb{N}^{n}\right\}, \\
\mathscr{L}^{\left(\mathbb{M}^{*}\right)}:= & \{Q \in \mathscr{L} \mid \text { for any } \varepsilon>0, \text { there exist } C, r>0 \text { such that } \\
& \left.\left\|b_{v}(z)\right\|_{\varepsilon} \leqslant C \frac{r^{|v|}}{M_{v}^{*}} \text { for all } v \in \mathbb{N}^{n}\right\} .
\end{aligned}
$$

Lemma 3. There exists $C>0$ such that for all $\beta \in \mathbb{N}^{n}, r>0$ and $f \in \mathbb{C}[[z]]$, we have

$$
\left\|f(z) z^{\beta}\right\|_{r t} \leqslant C\|f(z)\|_{r} \cdot \frac{M_{\beta}}{r^{|\beta|}} .
$$

Proof. By (M1), there exists $C>0$ such that for all $\beta \in \mathbb{N}^{n}, r>0$ and $f \in \mathbb{C}[[z]]$, we have that

$$
\begin{aligned}
\left\|f(z) z^{\beta}\right\|_{r t} & =\left\|\sum_{\alpha \in \mathbb{N}^{n}} f_{\alpha} z^{\alpha+\beta}\right\|_{r t}=\sup _{\alpha \in \mathbb{N}^{n}}\left|f_{\alpha}\right| \frac{M_{\alpha+\beta}}{(r t)^{|\alpha+\beta|}} \\
& \leqslant \sup _{\alpha \in \mathbb{N}^{n}}\left|f_{\alpha}\right| \frac{M_{\alpha}}{r^{|\alpha+\beta|}} \cdot \sup _{\alpha \in \mathbb{N}^{n}} \frac{M_{\alpha+\beta}}{M_{\alpha}}\left(\frac{1}{t}\right)^{|\alpha+\beta|} \leqslant C\|f(z)\|_{r} \cdot \frac{M_{\beta}}{r^{|\beta|}},
\end{aligned}
$$

as desired. 
By the following proposition, we see that the mapping $I: \mathscr{D}^{\left\{\mathbb{M}^{*}\right\}} \rightarrow \mathscr{L}^{\left\{\mathbb{M}^{*}\right\}}$ is bijective.

Proposition 3. $P \in \mathscr{D}^{\left\{\mathbb{M}^{*}\right\}}$ if and only if $I(P) \in \mathscr{L}^{\left\{\mathbb{M}^{*}\right\}}$.

Proof. (Necessity). For any $\varepsilon>0$, we choose some $0<\delta<\varepsilon / t$. Since $P \in \mathscr{D}^{\left\{\mathbb{M}^{*}\right\}}$, we have that there exist $C_{1}>0$ and $\delta+1 / r<\varepsilon / t$ such that

$$
\left\|a_{\alpha}(z)\right\|_{r} \leqslant C_{1} \delta^{|\alpha|} \frac{M_{\alpha}}{\alpha !}
$$

for all $\alpha \in \mathbb{N}^{n}$. By Lemma 2 (2) and Lemma 3, we have that there exist $C_{1}<C_{2}<C_{3}<C$ such that

$$
\begin{aligned}
\left\|b_{v}(z)\right\|_{r t} \frac{v !}{M_{v}} & \leqslant \sum_{\alpha \leqslant v} \frac{v !}{(v-\alpha) ! M_{v}}\left\|a_{\alpha}(z) z^{v-\alpha}\right\|_{r t} \\
& \leqslant \sum_{\alpha \leqslant v} \frac{C_{2} v !}{(v-\alpha) ! M_{v}} \cdot\left\|a_{\alpha}(z)\right\|_{r} \cdot \frac{M_{v-\alpha}}{r^{|v-\alpha|}} \\
& \leqslant \sum_{\alpha \leqslant v} \frac{C_{3} v !}{(v-\alpha) ! M_{v}} \cdot \frac{M_{\alpha}}{\alpha !} \delta^{|\alpha|} \cdot \frac{M_{v-\alpha}}{r^{|v-\alpha|}} \\
& \leqslant C t^{|v|} \sum_{\alpha \leqslant v}\left(\begin{array}{l}
v \\
\alpha
\end{array}\right) \frac{\delta^{|\alpha|}}{r^{|v-\alpha|}}=C t^{|v|}\left(\delta+\frac{1}{r}\right)^{|v|}<C \varepsilon^{|v|}
\end{aligned}
$$

for all $v \in \mathbb{N}^{n}$, which means $I(P) \in \mathscr{L}^{\left\{\mathbb{M}^{*}\right\}}$.

(Sufficiency). If $I(P) \in \mathscr{L}^{\left\{\mathbb{M}^{*}\right\}}$, then Lemma 2 (3) holds. By the same process as above, we have $P \in \mathscr{D}^{\left\{\mathbb{M}^{*}\right\}}$.

Let $P \in \mathscr{D}^{\left\{\mathbb{M}^{*}\right\}}$. To characterize continuous endomorphisms of $\mathscr{F}\{\mathbb{M}\}$, we need to show that the definition of $P f$ is unambiguous for all $f \in \mathscr{F}\{\mathbb{M}\}$.

Lemma 4. Every element of $\mathscr{D}^{\left\{\mathbb{M}^{*}\right\}}$ is formally well-defined on $\mathscr{F}\{\mathbb{M}\}$.

Proof. Suppose $f(z):=\sum_{v \in \mathbb{N}^{n}} f_{v} z^{v} \in \mathscr{F}\{\mathbb{M}\}$. Then we have that there exist $C_{1}, \delta>1$ such that

$$
\left|f_{v}\right| \leqslant C_{1} \frac{\delta^{|v|}}{M_{v}}
$$

for all $v \in \mathbb{N}^{n}$. If $P \in \mathscr{D}^{\left\{\mathbb{M}^{*}\right\}}$, then we have that for any $\varepsilon>0$, there exist $C_{2}, r>1$ such that

$$
\left|a_{\alpha}^{\beta}\right| \leqslant C_{2} \frac{M_{\alpha}}{\alpha ! M_{\beta}} \varepsilon^{|\alpha|} r^{|\beta|}
$$


for all $\alpha, \beta \in \mathbb{N}^{n}$. To see $P$ is formally well-defined at $f$, we choose some $\varepsilon>0$ such that $0<2 \varepsilon \delta t<1$. By Lemma $2(4)$, there exist $C>C_{3}>0$ such that for all $\mu \in \mathbb{N}^{n}$, we have

$$
\begin{aligned}
& \sum_{v \in \mathbb{N}^{n}} \sum_{\substack{\lambda \leqslant v \\
\lambda \leqslant \mu}}\left|a_{v-\lambda}^{\mu-\lambda} \frac{v !}{\lambda !} f_{v}\right| \leqslant\left. C_{1} C_{2} \sum_{v \in \mathbb{N}^{n}} \sum_{\substack{\lambda \leqslant v \\
\lambda \leqslant \mu}} \frac{M_{v-\lambda}}{(v-\lambda) ! M_{\mu-\lambda}} \varepsilon^{|v-\lambda|}\right|^{|\mu-\lambda|} \cdot \frac{v !}{\lambda !} \cdot \frac{\delta^{|v|}}{M_{v}} \\
& \leqslant \frac{C_{1} C_{2}}{M_{\mu}}\left(\begin{array}{l}
r \\
\varepsilon
\end{array}\right)^{|\mu|} \sum_{v \in \mathbb{N}^{n}}(\varepsilon \delta)^{|v|} \sum_{\substack{\lambda \leqslant v \\
\lambda \leqslant \mu}}\left(\begin{array}{l}
v \\
\lambda
\end{array}\right) \frac{M_{v-\lambda} M_{\lambda}}{M_{v}} \cdot \frac{M_{\mu}}{M_{\lambda} M_{\mu-\lambda}} \\
& \leqslant \frac{C_{3}}{M_{\mu}}\left(\frac{r t}{\varepsilon}\right)^{|\mu|} \sum_{\nu \in \mathbb{N}^{n}}(\varepsilon \delta t)^{|v|} \sum_{\substack{\lambda \leqslant v \\
\lambda \leqslant \mu}}\left(\begin{array}{l}
v \\
\lambda
\end{array}\right) \\
& \leqslant \frac{C_{3}}{M_{\mu}}\left(\frac{r t}{\varepsilon}\right)^{|\mu|} \sum_{v \in \mathbb{N}^{n}}(2 \varepsilon \delta t)^{|v|} \leqslant \frac{C}{M_{\mu}}\left(\frac{r t}{\varepsilon}\right)^{|\mu|}<\infty,
\end{aligned}
$$

as desired.

THEOREM 1 (Main Result 1). If $P \in \mathscr{D}^{\left\{\mathbb{M}^{*}\right\}}$ or $I(P) \in \mathscr{L}^{\left\{\mathbb{M}^{*}\right\}}$, then $P: \mathscr{F}\{\mathbb{M}\} \rightarrow \mathscr{F}\{\mathbb{M}\}$ is a continuous endomorphism.

Conversely, if $F: \mathscr{F}^{\{\mathbb{M}\}} \rightarrow \mathscr{F}^{\{\mathbb{M}\}}$ is a continuous linear operator, then we have

(1) there exists a unique $P \in \mathscr{D}^{\left\{\mathbb{M}^{*}\right\}}$ such that $F f=$ Pf for all $f \in \mathscr{F}\{\mathbb{M}\}$,

(2) there exists a unique $Q \in \mathscr{L}^{\left\{\mathbb{M}^{*}\right\}}$ such that $F f=Q f$ for all $f \in \mathscr{F}\{\mathbb{M}\}$ ',

Proof. In view of Proposition 3, we may assume both of $P \in \mathscr{D}^{\left\{\mathbb{M}^{*}\right\}}$ and $I(P) \in \mathscr{L}^{\left\{\mathbb{M}^{*}\right\}}$ hold. For any $\delta>0$, we choose some $0<\varepsilon<1 / \delta$. By Lemma 4 and Proposition 2, we have that there exist $h>0$ and $C>C_{1}>0$ such that

$$
\begin{aligned}
\|P f\|_{h}=\|I(P) f\|_{h} & \leqslant \sum_{v \in \mathbb{N}^{n}}\left\|b_{v}(z) \partial_{z}^{v} f(0)\right\|_{h} \\
& \leqslant \sum_{v \in \mathbb{N}^{n}}\left\|b_{v}(z)\right\|_{h} v !\left|f_{v}\right| \\
& \leqslant \sum_{v \in \mathbb{N}^{n}} C_{1} \varepsilon^{|v|} \frac{M_{v}}{v !} \cdot v ! \cdot\|f(z)\|_{\delta} \frac{\delta^{|v|}}{M_{v}} \leqslant C\|f(z)\|_{\delta}
\end{aligned}
$$

for all $f \in \mathscr{F}\{\mathbb{M}\}$.

To see the converse part, in view of Proposition 3, Lemma 4, and Proposition 2, it suffices to show (2). Since $F\left(z^{v}\right) \in \mathbb{C}[[z]]$ for all $v \in \mathbb{N}^{n}$, 
we have

$$
Q:=\sum_{v \in \mathbb{N}^{n}} F\left(\frac{z^{v}}{v !}\right) \partial_{z=0}^{v} \in \mathscr{L} .
$$

Hence, for any $f \in \mathscr{F}^{\{\mathbb{M}\}}$, we have

$$
F f=\sum_{v \in \mathbb{N}^{n}} f_{v} F\left(z^{v}\right)=\sum_{v \in \mathbb{N}^{n}} F\left(\frac{z^{v}}{v !}\right) \partial_{z}^{v} f(0)=Q f .
$$

It is obvious that $Q \in \mathscr{L}$ is unique.

Finally, we show $Q \in \mathscr{L}^{\left\{\mathbb{M}^{*}\right\}}$. For any $\varepsilon>0$, we choose some $h>1 / \varepsilon$. By the continuity of $F$, we have that there exist $r>h$ and $C>0$ such that

$$
\left\|F\left(\frac{z^{v}}{v !}\right)\right\|_{r} \leqslant \frac{C}{v !}\left\|z^{v}\right\|_{h}=\frac{C}{v !} \cdot \frac{M_{v}}{h^{|v|}} \leqslant C \frac{\varepsilon^{|v|}}{M_{v}^{*}}
$$

for all $v \in \mathbb{N}^{n}$, which implies $Q \in \mathscr{L}^{\left\{\mathbb{M}^{*}\right\}}$.

\section{Continuous endomorphisms of $\mathscr{F}^{(\mathbb{M})}$}

By the following proposition, we see that the mapping $I: \mathscr{D}^{\left(\mathbb{M}^{*}\right)} \rightarrow \mathscr{L}^{\left(\mathbb{M}^{*}\right)}$ is bijective.

Proposition 4. $\quad P \in \mathscr{D}^{\left(\mathbb{M}^{*}\right)}$ if and only if $I(P) \in \mathscr{L}^{\left(\mathbb{M}^{*}\right)}$.

Proof. (Necessity). Since $P \in \mathscr{D}^{\left(\mathbb{M}^{*}\right)}$, we have that for any $\varepsilon>0$, there exist $C_{1}, \delta>0$ such that

$$
\left\|a_{\alpha}(z)\right\|_{\varepsilon / t} \leqslant C_{1} \delta^{|\alpha|} \frac{M_{\alpha}}{\alpha !}
$$

for all $\alpha \in \mathbb{N}^{n}$. Thus, by Lemma 2 (2) and Lemma 3, we have that there exist $C_{1}<C_{2}<C_{3}<C$ such that

$$
\begin{aligned}
\left\|b_{v}(z)\right\|_{\varepsilon} \frac{v !}{M_{v}} & \leqslant \sum_{\alpha \leqslant v} \frac{v !}{(v-\alpha) ! M_{v}}\left\|a_{\alpha}(z) z^{v-\alpha}\right\|_{\varepsilon} \\
& \leqslant \sum_{\alpha \leqslant v} \frac{C_{2} v !}{(v-\alpha) ! M_{v}} \cdot\left\|a_{\alpha}(z)\right\|_{\varepsilon / t} \cdot \frac{M_{v-\alpha}}{(\varepsilon / t)^{|v-\alpha|}} \\
& \leqslant \sum_{\alpha \leqslant v} \frac{C_{3} v !}{(v-\alpha) ! M_{v}} \cdot \frac{M_{\alpha}}{\alpha !} \delta^{|\alpha|} \cdot M_{v-\alpha}\left(\frac{t}{\varepsilon}\right)^{|v-\alpha|} \\
& \leqslant C t^{|v|} \sum_{\alpha \leqslant v}\left(\begin{array}{l}
v \\
\alpha
\end{array}\right) \delta^{|\alpha|}\left(\frac{t}{\varepsilon}\right)^{|v-\alpha|}=C t^{|v|}\left(\delta+\frac{t}{\varepsilon}\right)^{|v|}
\end{aligned}
$$

for all $v \in \mathbb{N}^{n}$, which means $I(P) \in \mathscr{L}^{\left(\mathbb{M}^{*}\right)}$. 
(Sufficiency). If $I(P) \in \mathscr{L}^{\left(\mathbb{M}^{*}\right)}$, then Lemma 2 (3) holds. By the same process as above, we have $P \in \mathscr{D}^{\left(\mathbb{M}^{*}\right)}$.

Let $P \in \mathscr{D}^{\left(\mathbb{M}^{*}\right)}$. To characterize continuous endomorphisms of $\mathscr{F}(\mathbb{M})$, we need to show that the definition of $P f$ is unambiguous for all $f \in \mathscr{F}^{(\mathbb{M})}$.

Lemma 5. Every element of $\mathscr{D}^{\left(\mathbb{M}^{*}\right)}$ is formally well-defined on $\mathscr{F}^{(\mathbb{M})}$.

Proof. If $P \in \mathscr{D}^{\left(\mathbb{M}^{*}\right)}$, then we have that for any $0<\varepsilon<1$, there exist $C_{2}, r>0$ such that

$$
\left|a_{\alpha}^{\beta}\right| \leqslant C_{2} \frac{M_{\alpha}}{\alpha ! M_{\beta}} r^{|\alpha|} \varepsilon^{|\beta|}
$$

for all $\alpha, \beta \in \mathbb{N}^{n}$. If $f(z):=\sum_{v \in \mathbb{N}^{n}} f_{v} z^{v} \in \mathscr{F}^{(\mathbb{M})}$, then we have that for any $\delta>0$, there exists $C_{1}>0$ such that

$$
\left|f_{v}\right| \leqslant C_{1} \frac{\delta^{|v|}}{M_{v}}
$$

for all $v \in \mathbb{N}^{n}$. To see $P$ is formally well-defined at $f$, we choose some $\delta>0$ such that $0<\delta t(1+r)<1$. By Lemma $2(4)$, there exist $C>C_{3}>0$ such that for all $\mu \in \mathbb{N}^{n}$, we have

$$
\begin{aligned}
& \sum_{v \in \mathbb{N}^{n}} \sum_{\substack{\lambda \leqslant v \\
\lambda \leqslant \mu}}\left|a_{v-\lambda}^{\mu-\lambda} \frac{v !}{\lambda !} f_{v}\right| \leqslant C_{1} C_{2} \sum_{v \in \mathbb{N}^{n}} \sum_{\substack{\lambda \leqslant v \\
\lambda \leqslant \mu}} \frac{M_{v-\lambda}}{(v-\lambda) ! M_{\mu-\lambda}} r^{|v-\lambda|} \varepsilon^{|\mu-\lambda|} \cdot \frac{v !}{\lambda !} \cdot \frac{\delta^{|v|}}{M_{v}} \\
& \leqslant \frac{C_{1} C_{2}}{M_{\mu}} \sum_{v \in \mathbb{N}^{n}} \delta^{|v|} \sum_{\substack{\lambda \leqslant v \\
\lambda \leqslant \mu}}\left(\begin{array}{l}
v \\
\lambda
\end{array}\right) r^{|v-\lambda|} \frac{M_{v-\lambda} M_{\lambda}}{M_{v}} \cdot \frac{M_{\mu}}{M_{\lambda} M_{\mu-\lambda}} \\
& \leqslant \frac{C_{3} t^{|\mu|}}{M_{\mu}} \sum_{v \in \mathbb{N}^{n}}(\delta t)^{|v|} \sum_{\substack{\lambda \leqslant v \\
\lambda \leqslant \mu}}\left(\begin{array}{l}
v \\
\lambda
\end{array}\right) r^{|v-\lambda|} \\
& \leqslant \frac{C_{3} t^{|\mu|}}{M_{\mu}} \sum_{v \in \mathbb{N}^{n}}(\delta t(1+r))^{|v|} \leqslant \frac{C t^{|\mu|}}{M_{\mu}}<\infty
\end{aligned}
$$

as desired.

THEOREM 2 (Main Result 2). If $P \in \mathscr{D}^{\left(\mathbb{M}^{*}\right)}$ or $I(P) \in \mathscr{L}^{\left(\mathbb{M}^{*}\right)}$, then $P: \mathscr{F}^{(\mathbb{M})} \rightarrow \mathscr{F}^{(\mathbb{M})}$ is a continuous endomorphism.

Conversely, if $F: \mathscr{F}^{(\mathbb{M})} \rightarrow \mathscr{F}^{(\mathbb{M})}$ is a continuous linear operator, then we have 
(1) there exists a unique $P \in \mathscr{D}^{\left(\mathbb{M}^{*}\right)}$ such that $F f=$ Pf for all $f \in \mathscr{F}^{(\mathbb{M})}$,

(2) there exists a unique $Q \in \mathscr{L}^{\left(\mathbb{M}^{*}\right)}$ such that $F f=Q f$ for all $f \in \mathscr{F}^{(\mathbb{M})}$ '.

Proof. In view of Proposition 4, we may assume both of $P \in \mathscr{D}^{\left(\mathbb{M}^{*}\right)}$ and $I(P) \in \mathscr{L}^{\left(\mathbb{M}^{*}\right)}$ hold. By the definition of $\mathscr{L}^{\left(\mathbb{M}^{*}\right)}$, we have that for any $\varepsilon>0$, there exist $C_{1}, r>0$ such that

$$
\left\|b_{v}(z)\right\|_{\varepsilon} \leqslant C_{1} r^{|v|} \frac{M_{v}}{v !}
$$

for all $v \in \mathbb{N}^{n}$. We choose some $0<\delta<1 / r$. By Lemma 4 and Proposition 2, we have that there exist $h>0$ and $C>C_{1}$ such that

$$
\begin{aligned}
\|P f\|_{\varepsilon}=\|I(P) f\|_{\varepsilon} & \leqslant \sum_{v \in \mathbb{N}^{n}}\left\|b_{v}(z) \partial_{z}^{v} f(0)\right\|_{\varepsilon} \\
& \leqslant \sum_{v \in \mathbb{N}^{n}}\left\|b_{v}(z)\right\|_{\varepsilon} v !\left|f_{v}\right| \\
& \leqslant \sum_{v \in \mathbb{N}^{n}} C_{1} r^{|v|} \frac{M_{v}}{v !} \cdot v ! \cdot\|f(z)\|_{\delta} \frac{\delta^{|v|}}{M_{v}} \leqslant C\|f(z)\|_{\delta}
\end{aligned}
$$

for all $f \in \mathscr{F}^{(\mathbb{M})}$.

To see the converse part, in view of Proposition 4, Lemma 4, and Proposition 2, it suffices to show (2). Considering the proof of the converse part of Theorem 1, we only need to prove that the operator $Q$ defined in the proof of Theorem 1 is in $\mathscr{L}^{\left(\mathbb{M}^{*}\right)}$. By the continuity of $F$, we have that for any $\varepsilon>0$, there exist $C, h>0$ and $r>1 / h$ such that

$$
\left\|F\left(\frac{z^{v}}{v !}\right)\right\|_{\varepsilon} \leqslant \frac{C}{v !}\left\|z^{v}\right\|_{h}=\frac{C}{v !} \cdot \frac{M_{v}}{h^{|v|}} \leqslant C \frac{r^{|v|}}{M_{v}^{*}}
$$

for all $v \in \mathbb{N}^{n}$, which implies $Q \in \mathscr{L}^{\left(\mathbb{M}^{*}\right)}$.

\section{Applications}

We conclude this paper by applying the main results to the space of entire functions of normal type or minimal type with respect to a proximate order. In this section, we assume $\rho>0$. Let $\rho(r)$ be a proximate order for the order $\rho$ (see Definition 1.15, [9]). We recall that $\lim _{r \rightarrow \infty} \rho(r)=\rho$. For any $\sigma>0$, we define the Banach space

$$
B_{w_{\sigma}}:=\left\{f \in \mathcal{O}\left(\mathbb{C}^{n}\right)\left|\|f\|_{w_{\sigma}}:=\sup _{z \in \mathbb{C}^{n}}\right| f(z) \mid \mathrm{e}^{-w_{\sigma}(z)}<+\infty\right\}
$$


with norm $\|\cdot\|_{w_{\sigma}}$, where $w_{\sigma}:=\sigma|z|^{\rho(|z|)}$. Now we consider the space of entire functions of normal type with respect to a proximate order $\rho(r)$ :

$$
\mathscr{E}^{\rho(r)}:=\lim _{\sigma \rightarrow \infty} B_{w_{\sigma}}
$$

which is a (DFS)-space (see [6]), and the space of entire functions of minimal type with respect to a proximate order $\rho(r)$ :

$$
E_{0}^{\rho(r)}:=\lim _{\sigma \rightarrow 0} B_{w_{\sigma}}
$$

which is an (FS)-space. Let $\varphi(q)$ be the inverse function of $q=r^{\rho(r)}$ for all sufficiently large $q \in \mathbb{R}$. It's well-known that $r^{\rho(r)}$ is strictly increasing for all sufficiently large $r>0$. We may assume the function $\varphi(q)$ is strictly increasing on $q \in[0, \infty)$. Let $\mathbb{A}:=\left(A_{|\alpha|}\right)_{\alpha \in \mathbb{N}^{n}}$ and $\mathbb{A}^{*}:=\left(A_{|\alpha|}^{*}\right)_{\alpha \in \mathbb{N}^{n}}$, where

$$
A_{|\alpha|}:=\frac{\varphi(|\alpha|)^{|\alpha|}}{(\mathrm{e} \rho)^{|\alpha| / \rho}}
$$

and $A_{|\alpha|}^{*}:=\alpha ! / A_{|\alpha|}$. We remark that (see Proposition $1[6]$ and Proposition 3.3 $[7])$ :

Proposition 5. Suppose that $f(z)=\sum_{\alpha \in \mathbb{N}^{n}} f_{\alpha} z^{\alpha}$ is an entire function. Then,

(1) $f(z)$ belongs to $\mathscr{E}^{\rho(r)}$ if and only if we have

$$
\limsup _{|\alpha| \rightarrow \infty}\left(\left|f_{\alpha}\right| A_{|\alpha|}\right)^{1 /|\alpha|}<\infty
$$

(2) $f(z)$ belongs to $E_{0}^{\rho(r)}$ if and only if we have

$$
\limsup _{|\alpha| \rightarrow \infty}\left(\left|f_{\alpha}\right| A_{|\alpha|}\right)^{1 /|\alpha|}=0 .
$$

Therefore, we see $\mathscr{F}^{\{\mathbb{A}\}}=\mathscr{E}^{\rho(r)}$ and $\mathscr{F}^{(\mathbb{A})}=E_{0}^{\rho(r)}$ in the sense of set because of the following lemma.

Lemma 6. The sequence $\mathbb{A}$ satisfies (M1).

Proof. We remark that (see the proof of Theorem 1.23 [9]) for any $0<\varepsilon<1 / \rho$, there exists $S_{\varepsilon}>0$ such that

$$
\left(\frac{1}{\rho}-\varepsilon\right) \frac{\mathrm{d}}{\mathrm{d} s} \log s<\frac{\mathrm{d}}{\mathrm{d} s} \log \varphi(s)<\left(\frac{1}{\rho}+\varepsilon\right) \frac{\mathrm{d}}{\mathrm{d} s} \log s
$$


whenever $s>S_{\varepsilon}$. Set $\xi:=1 / \rho+\varepsilon$. For any $p, q>S_{\varepsilon}$, integrating each side from $p$ to $p+q$, we have

$$
\left(\frac{1}{\rho}-\varepsilon\right) \log \frac{p+q}{p}<\log \frac{\varphi(p+q)}{\varphi(p)}<\left(\frac{1}{\rho}+\varepsilon\right) \log \frac{p+q}{p} .
$$

Hence, for all $p, q>S_{\varepsilon}$, we have that

$$
\left(\frac{p+q}{p}\right)^{\left(\rho^{-1}-\varepsilon\right) p}<\left(\frac{\varphi(p+q)}{\varphi(p)}\right)^{p}<\left(\frac{p+q}{p}\right)^{\left(\rho^{-1}+\varepsilon\right) p} .
$$

By the same reason, for all $p, q>S_{\varepsilon}$, we have that

$$
\left(\frac{p+q}{q}\right)^{\left(\rho^{-1}-\varepsilon\right) q}<\left(\frac{\varphi(p+q)}{\varphi(q)}\right)^{q}<\left(\frac{p+q}{q}\right)^{\left(\rho^{-1}+\varepsilon\right) q} .
$$

Therefore, for all $p, q>S_{\varepsilon}$, we have that

$$
\begin{aligned}
\frac{A_{p+q}}{A_{p} A_{q}}=\frac{\varphi(p+q)^{p+q}}{\varphi(p)^{p} \varphi(q)^{q}} & =\left(\frac{\varphi(p+q)}{\varphi(p)}\right)^{p}\left(\frac{\varphi(p+q)}{\varphi(q)}\right)^{q} \\
& \leqslant\left(\frac{p+q}{p}\right)^{\left(\rho^{-1}+\varepsilon\right) p}\left(\frac{p+q}{q}\right)^{\left(\rho^{-1}+\varepsilon\right) q}<\mathrm{e}^{\left(\rho^{-1}+\varepsilon\right)(p+q)}
\end{aligned}
$$

and

$$
\begin{aligned}
\frac{A_{p} A_{q}}{A_{p+q}}=\frac{\varphi(p)^{p} \varphi(q)^{q}}{\varphi(p+q)^{p+q}} & =\left(\frac{\varphi(p)}{\varphi(p+q)}\right)^{p}\left(\frac{\varphi(q)}{\varphi(p+q)}\right)^{q} \\
& \leqslant\left(\frac{p}{p+q}\right)^{\left(\rho^{-1}-\varepsilon\right) p}\left(\frac{q}{p+q}\right)^{\left(\rho^{-1}-\varepsilon\right) q}<1
\end{aligned}
$$

And the assertion follows immediately.

We recall an important lemma (see Lemma 6.3 [7]):

Lemma 7. Assume that, for each $q \in \mathbb{Z}_{+}, r:=r(q)$ is the solution of equation

$$
\frac{\mathrm{d}}{\mathrm{d} r}\left(r^{q} \mathrm{e}^{-\sigma r^{\rho(r)}}\right)=0
$$

Then we have that

$$
\lim _{q \rightarrow \infty}\left(\frac{r^{q} \mathrm{e}^{-\sigma r^{\rho(r)}}}{A_{q}}\right)^{1 / q}=\left(\frac{1}{\sigma}\right)^{1 / \rho}
$$


The following lemma shows that the topology of $\mathscr{E}^{\rho(r)}$ (resp., $E_{0}^{\rho(r)}$ ) coincides with the topology of $\mathscr{F}^{\{\mathbb{A}\}}$ (resp., $\mathscr{F}^{(\mathbb{A})}$ ), that is $\mathscr{F}^{\{\mathbb{A}\}}=\mathscr{E}^{\rho(r)}$ (resp., $\left.\mathscr{F}^{(\mathbb{A})}=E_{0}^{\rho(r)}\right)$ in the sense of topological spaces.

Lemma 8. Suppose $\rho>0$ and $M_{\alpha}=A_{|\alpha|}$ for all $\alpha \in \mathbb{N}^{n}$. Then

(1) For any $\delta>0$, there exist $\sigma, C>0$ such that for all $f \in B_{\delta}^{\mathbb{M}}$, we have

$$
\frac{\|f\|_{\delta}}{C} \leqslant\|f\|_{w_{\sigma}} \leqslant C\|f\|_{\delta}
$$

(2) For any $\sigma>0$, there exist $\delta, C>0$ such that for all $f \in B_{w_{\sigma}}$, we have

$$
\frac{\|f\|_{w_{\sigma}}}{C} \leqslant\|f\|_{\delta} \leqslant C\|f\|_{w_{\sigma}}
$$

Proof. Let $\vec{S}:=(s, \ldots, s) \in \mathbb{R}_{+}^{n}$ and $r:=s \sqrt{n}$. By the Cauchy inequality, for any $s>0$ and any $\sigma>0$, we have

$$
\begin{aligned}
\left|f_{\alpha}\right|=\frac{1}{\alpha !}\left|\partial_{z}^{\alpha} f(0)\right| & \leqslant \frac{1}{\vec{S}^{\alpha}} \sup _{|\vec{z}| \leqslant \vec{S}}|f(z)| \leqslant\left(\frac{1}{s}\right)^{|\alpha|} \sup _{|z| \leqslant s \sqrt{n}}|f(z)| \\
& =\left(\frac{\sqrt{n}}{r}\right)^{|\alpha|}\left(\sup _{|z| \leqslant r}|f(z)| \mathrm{e}^{-\sigma r^{\rho(r)}}\right) \mathrm{e}^{\sigma r^{\rho(r)}} \\
& \leqslant \frac{\|f\|_{w_{\sigma}}}{A_{|\alpha|}}(\sqrt{n})^{|\alpha|} \frac{A_{|\alpha|} \mathrm{e}^{\sigma r^{\rho(r)}}}{r^{|\alpha|}} .
\end{aligned}
$$

For each $|\alpha| \in \mathbb{Z}_{+}$, we choose some $r>0$ satisfying (6.2). Continuing the estimate, by Lemma 7, we have that for any $\varepsilon>0$, there exists $C>0$ such that

$$
\left|f_{\alpha}\right| \leqslant C \frac{\|f\|_{w_{\sigma}}}{A_{|\alpha|}}\left(\sqrt{n} \sigma^{1 / \rho}+\varepsilon\right)^{|\alpha|}
$$

for all $\alpha \in \mathbb{N}^{n}$. To see the first inequality in (1) (resp., the second inequality in (2)), for any $\delta>0$ (resp., $\sigma>0$ ), we choose some $\varepsilon>0$ and $\sigma>0$ (resp., $\delta>0$ ) such that $\sqrt{n} \sigma^{1 / \rho}+\varepsilon<\delta$. Hence, there exists $C>0$ such that

$$
\|f\|_{\delta}=\sup _{\alpha \in \mathbb{N}^{n}}\left|f_{\alpha}\right| \frac{A_{|\alpha|}}{\delta^{|\alpha|}} \leqslant \sup _{\alpha \in \mathbb{N}^{n}}\left|f_{\alpha}\right| A_{|\alpha|}\left(\sqrt{n} \sigma^{1 / \rho}+\varepsilon\right)^{-|\alpha|} \leqslant C\|f\|_{w_{\sigma}}
$$

for all $f \in B_{\delta}^{\mathbb{M}}$ (resp., $f \in B_{w_{\sigma}}$ ). 
To see the second inequality in (1) (resp., the first inequality in (2)), we remark that (see the proof of Lemma 6.4 in [7]) if $\rho, \sigma>0$, then

$$
\limsup _{|\alpha| \rightarrow \infty}\left(\frac{\left\|z^{\alpha}\right\|_{w_{\sigma}}}{A_{|\alpha|}}\right)^{1 /|\alpha|} \leqslant\left(\frac{1}{\sigma}\right)^{1 / \rho} .
$$

For any $\delta>0$ (resp., $\sigma>0$ ), we choose some $\varepsilon>0$ and $\sigma>0$ (resp., $\delta>0$ ) such that $\delta\left((1 / \sigma)^{1 / \rho}+\varepsilon\right)<1$. Then there exists $C>0$ such that

$$
\begin{aligned}
\|f\|_{w_{\sigma}} \leqslant \sum_{\alpha \in \mathbb{N}^{n}}\left|f_{\alpha}\right|\left\|z^{\alpha}\right\|_{w_{\sigma}} & \leqslant \sum_{\alpha \in \mathbb{N}^{n}}\left|f_{\alpha}\right| A_{|\alpha|}\left(\left(\frac{1}{\sigma}\right)^{1 / \rho}+\varepsilon\right)^{|\alpha|} \\
& \leqslant\|f\|_{\delta} \sum_{\alpha \in \mathbb{N}^{n}} \delta^{|\alpha|}\left(\left(\frac{1}{\sigma}\right)^{1 / \rho}+\varepsilon\right)^{|\alpha|} \leqslant C\|f\|_{\delta}
\end{aligned}
$$

for all $f \in B_{\delta}^{\mathbb{M}}$ (resp., $f \in B_{w_{\sigma}}$ ), as desired.

Finally, applying the preceding lemma and the main results, we have the following corollaries immediately.

Corollary 1. Let $P \in \mathscr{D}$ and assume that one of the following conditions holds:

(i) $P \in \mathscr{D}^{\left\{\mathbb{A}^{*}\right\}}$.

(ii) $I(P) \in \mathscr{L}^{\left\{\mathbb{A}^{*}\right\}}$.

(iii) For any $\varepsilon>0$, there exist $C, \sigma>0$ such that for all $\alpha \in \mathbb{N}^{n}$, we have

$$
\left\|a_{\alpha}(z)\right\|_{w_{\sigma}} \leqslant C \frac{\varepsilon^{|\alpha|}}{A_{|\alpha|}^{*}} .
$$

(iv) For any $\varepsilon>0$, there exist $C, \sigma>0$ such that for all $v \in \mathbb{N}^{n}$, we have

$$
\left\|P\left(\frac{z^{v}}{v !}\right)\right\|_{w_{\sigma}} \leqslant C \frac{\varepsilon^{|v|}}{A_{|v|}^{*}} .
$$

Then $P: \mathscr{E}^{\rho(r)} \rightarrow \mathscr{E}^{\rho(r)}$ is a continuous endomorphism.

Conversely, if $F: \mathscr{E}^{\rho(r)} \rightarrow \mathscr{E}^{\rho(r)}$ is a continuous linear operator, then we have

(1) There exists a unique $P \in \mathscr{D}$ satisfying condition (iii) or (iv) such that $F f=P f$ for all $f \in \mathscr{E}^{\rho(r)}$.

(2) There exists a unique $P \in \mathscr{D}^{\left\{\mathbb{A}^{*}\right\}}$ such that $F f=$ Pf for all $f \in \mathscr{E} \rho(r)$.

(3) There exists a unique $Q \in \mathscr{L}^{\left\{\mathbb{A}^{*}\right\}}$ such that $F f=Q f$ for all $f \in \mathscr{E} \rho(r)$. 
Corollary 2. Let $P \in \mathscr{D}$ and assume that one of the following conditions holds:

(i) $P \in \mathscr{D}^{\left(\mathbb{A}^{*}\right)}$.

(ii) $I(P) \in \mathscr{L}^{\left(\mathbb{A}^{*}\right)}$.

(iii) For any $\varepsilon>0$, there exist $C, \delta>0$ such that for all $\alpha \in \mathbb{N}^{n}$, we have

$$
\left\|a_{\alpha}(z)\right\|_{w_{\varepsilon}} \leqslant C \frac{\delta^{|\alpha|}}{A_{|\alpha|}^{*}} .
$$

(iv) For any $\varepsilon>0$, there exist $C, \delta>0$ such that for all $v \in \mathbb{N}^{n}$, we have

$$
\left\|P\left(\frac{z^{v}}{v !}\right)\right\|_{w_{\varepsilon}} \leqslant C \frac{\delta^{|v|}}{A_{|v|}^{*}} .
$$

Then $P: E_{0}^{\rho(r)} \rightarrow E_{0}^{\rho(r)}$ is a continuous endomorphism.

Conversely, if $F: E_{0}^{\rho(r)} \rightarrow E_{0}^{\rho(r)}$ is a continuous linear operator, then we have

(1) There exists a unique $P \in \mathscr{D}$ satisfying condition (iii) or (iv) such that $F f=P f$ for all $f \in E_{0}^{\rho(r)}$.

(2) There exists a unique $P \in \mathscr{D}^{\left(\mathbb{A}^{*}\right)}$ such that $F f=P f$ for all $f \in E_{0}^{\rho(r)}$.

(3) There exists a unique $Q \in \mathscr{L}^{\left(\mathbb{A}^{*}\right)}$ such that $F f=Q f$ for all $f \in E_{0}^{\rho(r)}$.

In [2], continuous endomorphisms of the space $\mathscr{E}^{\rho(r)}\left(\right.$ resp., $\left.E_{0}^{\rho(r)}\right)$ are characterized by condition (iii) of Corollary 1 (resp., Corollary 2). Hence, these two corollaries could be considered as the extension of the theorems in $[2]$.

\section{References}

[1] T. Aoki, R. Ishimura, Y. Okada, D. C. Struppa and S. Uchida, Characterization of Continuous Endomorphisms in the Space of Entire Functions of a Given Order, arXiv:1805.00663v1 [math.FA].

[2] T. Aoki, R. Ishimura, Y. Okada, D. C. Struppa and S. Uchida, Characterization of Endomorphisms of the Space of Entire Functions for a Proximate Order, preprint.

[3] R. Ishimura, Homomorphismes du faisceau des germes de functions holomorphes dans lui-même et op'erateurs différentis, Mem. Fac. Sci. Kyushu Univ. 32 (1978), 301-312.

[4] R. Ishimura, Endomorphismes de l'espace des germes de fonctions holomorphes en un point et op'erateurs différentiels d'ordre infini, Ann. Polo. Math. 49 (1988), 129-133.

[5] R. Ishimura, Endomorphisms of the space of higher-order entire functions and infinite-order differential operators, Kyushu J. Math. 61 (2007), 83-94.

[6] R. Ishimura and X. Jin, Infinite order differential equations in the space of entire functions of normal type with respect to a proximate order, North-W. Eur. J. of Math. 5 (2019), 69-87. 
[7] X. Jin, Infinite order differential equations in the space of entire functions of minimal type with respect to a proximate order, preprint.

[8] H. Komatsu, Ultradistributions I, Structure theorems and a characterization, J. Fac. Sci. Univ. Tokyo, Sec. IA, 20 (1973), 25-105.

[9] P. Lelong and L. Gruman, Entire functions of several complex variables, Grung. Math. Wiss., Berlin, Hidelberg, New York, Springer vol. 282, 1986.

Xiaoran Jin

Graduate School of Science

Course of Mathematics and Informatics

Chiba University

Yayoicho, Chiba 263-8522, Japan

E-mail: jinxiaoran@gmail.com 